\title{
Práticas alimentares no primeiro ano de vida e fatores associados em amostra representativa da cidade de Porto Alegre, Rio Grande do Sul
}

\section{$(1$}

Feeding practices and associated factors in the first year of life in a representative sample of Porto Alegre, Rio Grande do Sul, Brazil

Paula Dal Bó CAMPAGNOLO'

Maria Laura da Costa LOUZADA²

Elizabeth Lemos SILVEIRA ${ }^{3}$

Márcia Regina VITOLO 4

RE S U M O

\section{Objetivo}

Investigar a adequação das práticas alimentares no primeiro ano de vida e seus fatores associados na cidade de Porto Alegre (RS).

\section{Métodos}

Este estudo transversal foi realizado durante a Campanha Nacional de Imunização no ano de 2008. Utilizou-se amostragem por conglomerados em dois estágios e foram avaliadas 1099 crianças menores de um ano de idade, em 31 postos de vacinação. A coleta de dados consistiu da aplicação de questionário estruturado sobre características maternas, uso de chupeta, consumo de leite materno, de leites artificiais, chá, água, alimentos complementares e de alimentos de baixo valor nutricional pelas crianças. Para estimar a associação entre variáveis maternas, uso de chupeta e práticas alimentares, foram utilizadas análises de regressão logística.

\section{Resultados}

A prevalência de aleitamento materno exclusivo foi de $47,1 \%$ entre as crianças com até quatro meses de vida e de $21,4 \%$ entre as crianças entre quatro e seis meses. A frequência de aleitamento materno exclusivo foi maior entre as crianças que não usavam chupeta, que não eram primogênitas e cujas mães não trabalhavam

\footnotetext{
1 Universidade do Vale do Rio dos Sinos, Centro de Ciências da Saúde. Av. Unisinos, 950, Cristo Rei, 90470-200, São Leopoldo, RS, Brasil. Correspondência para/Correspondence to: PDB CAMPAGNOLO. E-mail: <pcampagnolo@unisinos.br>.

2 Universidade Federal de Ciências da Saúde de Porto Alegre, Núcleo de Pesquisa em Nutrição. Porto Alegre, RS, Brasil.

3 Secretaria Municipal de Saúde de Porto Alegre. Porto Alegre, RS, Brasil.

${ }^{4}$ Universidade Federal de Ciências da Saúde de Porto Alegre, Departamento de Nutrição. Porto Alegre, RS, Brasil.
} 
432 | PDB CAMPAGNOLO et al.

fora de casa ou estavam em licença maternidade. Melhores práticas de alimentação complementar foram observadas em crianças cujas mães tinham maior nível de escolaridade e trabalhavam fora de casa.

\section{Conclusão}

Este estudo mostrou a existência de fatores de risco para a interrupção precoce do aleitamento materno exclusivo e o consumo de alimentos inadequados aos lactentes.

Termos de indexação: Aleitamento materno. Consumo de alimentos. Fatores de risco. Inquéritos nutricionais. Lactente.

\section{A B S T R A C T}

\section{Objective}

The present study aimed to investigate the adequacy of feeding practices in the first year of life and associated factors in the city of Porto Alegre (RS).

\section{Methods}

This cross-sectional study was performed during the national vaccination campaign in 2008. Two-stage cluster sampling was used and 1,099 children under one year of age were evaluated at 31 vaccination sites. Maternal and breastfeeding data and the use of pacifiers, infant formulas, tea, water, complementary foods and low-nutrient foods were investigated using a structured questionnaire. Logistic regression analyses were used to estimate the association between maternal characteristics, use of pacifiers and infant feeding practices.

\section{Results}

The prevalence of exclusive breastfeeding was $47.1 \%$ in children aged up to four months and $21.4 \%$ in children aged four to six months. The prevalence of exclusive breastfeeding was higher in children who did not use pacifiers, who were not firstborns and whose mothers were on maternity leave or not employed. Better complementary feeding practices were observed among employed mothers with higher education level.

\section{Conclusion}

This study evidenced the existence of risk factors for early discontinuation of exclusive breastfeeding and inappropriate feeding practices.

Indexing terms: Breast feeding. Food consumption. Risk factors. Nutrition Surveys. Infant.

\section{N T R O D U ÇÃ O}

A prática do aleitamento materno exclusivo por seis meses e a adequada introdução da alimentação complementar são diretrizes estabelecidas pelo Ministério da Saúde por meio dos "Dez passos para alimentação saudável para crianças brasileiras menores de dois anos" ${ }^{1}$. Há evidências de que os alimentos que a criança recebe nos primeiros dois anos de vida irão determinar suas preferências, que podem se prolongar até a vida adulta ${ }^{2-4}$. O período da introdução da alimentação complementar é relevante uma vez que a introdução de alimentos antes dos seis meses de vida aumenta a chance de as crianças apresentarem rejeição alimentar e neofobia ${ }^{5}$. Revisão sistemática recente também apontou que crianças que recebem fórmula infantil apresentam maior adiposidade quando comparadas às amamentadas ao peito ${ }^{6}$. Além disso, a maior ingestão de proteína estimula o Insulin-like Growth Factor 1 (IGF-1) e a insulina na infância7, levando ao aumento de alterações metabólicas na infância e na vida adulta.

Os dados de aleitamento materno no Brasil nas últimas pesquisas nacionais mostraram melhoras nessas práticas. A proporção de aleitamento materno exclusivo aos 2 e 3 meses aumentou de 26,4\% na pesquisa de 1996 para 48,3\% em 2006; na pesquisa de 2006, a prevalência de crianças amamentadas aos 12 meses de idade foi de 47,5\%, e aos 24 meses, de 24,8\%8. Dados coletados na campanha nacional de imunização em 2008 mostraram prevalências de aleitamento 
materno exclusivo de $41 \%$ de zero aos 6 meses e de aleitamento materno de $58,7 \%$ dos 9 aos 12 meses $^{9}$. O que se observa é que, apesar de todos os esforços que vêm sendo feitos no âmbito da política nacional nas últimas décadas, atingir os valores ideais quanto à duração do aleitamento materno exclusivo ainda é um desafio.

Dados nacionais recentes apontam que no quarto mês cerca de um terço das crianças recebia suco de fruta e um quarto das crianças recebia mingau, fruta ou sopa ${ }^{10}$, o que confirmou a introdução precoce dos alimentos complementares. Estudo anterior observou que o consumo diário de frutas, legumes e verduras, in natura ou em suco, foi relatado para 6 de cada 10 crianças a partir de 6 meses, e apenas 50\% consumiam carne entre 4 e 7 vezes na semana, e, para $10 \%$ delas, essa frequência era de apenas uma vez na semana ${ }^{8}$. Esses resultados evidenciam prevalência elevada de práticas inadequadas antes e depois dos 6 meses de vida em crianças brasileiras. Dessa forma, o presente estudo objetivou investigar a adequação das práticas alimentares no primeiro ano de vida e seus fatores associados na cidade de Porto Alegre (RS), utilizando os dados da pesquisa nacional realizada em 2008 durante a Campanha Nacional de Imunização.

\section{M É T O D O S}

Estudo transversal sobre práticas alimentares de amostra representativa da população de crianças menores de um ano de idade que compareceram aos postos de vacinação na segunda etapa da campanha da vacinação do ano de 2008 em Porto Alegre (RS). O cálculo amostral foi baseado nos dados da Coordenadoria Geral de Vigilância em Saúde, que descreveu a realização de 15403 vacinas em crianças menores de um ano na primeira etapa da campanha da vacinação nos 130 postos distribuídos nas 8 gerências distritais de Porto Alegre. Levou-se em consideração a prevalência de aleitamento materno exclusivo em menores de 6 meses em Porto Alegre em 1999, com acréscimo de 2 a $10 \%$, supondo aumento da prevalência entre 1999 e 2008. Acrescentou-se a taxa de não resposta, que variou entre 5\% e 10\%, resultando no tamanho amostral de 1099.

Adotou-se a amostragem por conglomerados sorteados em dois estágios para que a amostra de 1099 crianças fosse representativa da população de Porto Alegre. No primeiro estágio, foram determinados os postos nos quais seria realizada a coleta de dados pelos entrevistadores. Buscou-se a obtenção de amostra equiprobabilística, ou seja, as crianças vacinadas em grandes conglomerados tiveram maior chance de participar do primeiro sorteio e crianças vacinadas em pequenos conglomerados tiveram maior chance no segundo sorteio. Dessa forma, foram sorteados 31 postos (4 centros de saúde, 4 postos avançados, 8 equipes de saúde da família e 15 unidades básicas de saúde). No segundo estágio, foi realizada amostragem sistemática na fila de vacinas de acordo com critérios pré-estabelecidos pela equipe da coordenação nacional da pesquisa.

O questionário elaborado para coleta de dados evitou questões abertas para facilitar seu preenchimento e evitar que a realização das entrevistas prejudicasse o andamento da campanha de vacinação. As questões sobre alimentação referiram-se às últimas 24 horas; para facilitar a obtenção das informações, consideraram-se "últimas 24 horas" o que a criança recebeu da manhã do dia anterior à manhã do dia da pesquisa.

Cada posto de vacinas contemplado tinha pelo menos quatro entrevistadores e um supervisor de campo, todos treinados para realizarem as entrevistas, além de suporte da coordenação local (gerência distrital) e da coordenação municipal. A pesquisa estendeu-se por uma semana além do dia principal.

\section{Dados maternos}

Foram obtidos dados de escolaridade materna (categorizado em: sem e com ensino fundamental completo, ensino médio completo e ensino superior completo), ocupação materna no mo- 
mento da entrevista (categorizado em: trabalha fora, não trabalha fora e em licença maternidade) e em relação à paridade (categorizado em: primeiro filho - sim ou não).

\section{Dados das crianças}

Os responsáveis pelas crianças responderam a questões relacionadas ao consumo dos seguintes alimentos pela criança no dia anterior à entrevista: leite do peito, chá, água, outro leite, fruta, comida de panela/comida de sal, carne, feijão, legumes/verduras, fruta, suco de fruta, refrigerante, biscoito recheado/salgadinho, açúcar/ mel, café. Todas as variáveis foram categorizadas em consumiu e não consumiu. Foi perguntado se a criança havia usado chupeta/bico no dia anterior à entrevista.

O aleitamento materno foi classificado de acordo com os seguintes conceitos da Organização Pan-americana de Saúde e da Organização Mundial de Saúde: Aleitamento Materno Exclusivo (AME) - quando a criança recebe somente leite materno, diretamente da mama ou extraído, e nenhum outro líquido ou sólido, com exceção de gotas ou xaropes de vitaminas, minerais e/ou medicamentos; Aleitamento Materno Predominante (AMP) - quando o lactente recebe, além do leite materno, água ou bebidas à base de água, como sucos de frutas e chás; Aleitamento Materno (AM) - quando a criança recebe leite materno, diretamente do seio ou extraído, independentemente de estar recebendo qualquer alimento ou líquido, incluindo leite não humano ${ }^{11}$.

O projeto foi aprovado pelo Comitê de Ética em Pesquisa da Secretaria Municipal de Saúde de Porto Alegre (Processo nº 001.031882.08.0) e todos os responsáveis assinaram termo de consentimento livre e esclarecido. Este estudo não envolve conflito de interesses.

\section{Análise dos dados}

Foi utilizado um aplicativo desenvolvido pelo Serviço de Desenvolvimento de Sistemas \&
IS/SES/SP e Fundação Osvaldo Cruz (Fiocruz) para a digitação dos dados utilizando a Internet, por meio de sistema online (http://www.redeblh.icict. fiocruz.br/pesqam), que possui recursos de checagem dos dados durante a digitação. As análises foram realizadas no programa Statistical Package for the Social Sciences (SPSS) versão 16.0. A análise univariada expressou a frequência da variável de desfecho sob a forma de proporções. As análises da associação entre a proporção de crianças com menos de seis meses que estavam em aleitamento materno exclusivo no momento da entrevista e variáveis maternas, paridade e uso de chupeta foram expressas em Odds Ratio (OR), e seus respectivos Intervalos de Confiança de 95\% (IC95\%) e o valor de $p$ foi calculado por meio do teste Qui-quadrado. As variáveis que apresentaram $p<0,20$ na análise bivariada foram incluídas na análise de regressão logística. A associação entre o consumo de determinados alimentos no dia anterior à entrevista e dados de escolaridade e ocupação materna também foi expressa em OR e IC95\%. Foi considerada diferença estatisticamente significativa quando $p<0,05$.

\section{RES U LTA DOS}

Foram avaliadas 1099 crianças: 1087 apresentaram dados completos com relação ao aleitamento materno. Para as análises de associação do consumo de alimentos com as variáveis independentes, o número amostral utilizado variou conforme a quantidade de registros com os dados completos.

Entre as crianças avaliadas, 52,0\% eram do sexo masculino. Com relação à idade, 36,1\% tinham até quatro meses de vida, 16,6\% tinham entre quatro e seis meses de vida e 47,3\% acima de seis meses. A prevalência de crianças em aleitamento materno exclusivo no momento da entrevista foi de 47,1\% entre as crianças com até quatro meses de vida e $21,4 \%$ entre as crianças entre quatro e seis meses. A prevalência de aleitamento materno foi de $84,4 \%$ até quatro meses de vida e 73,6 entre quatro e seis meses. As prevalências de crianças que estavam recebendo outro 
leite que não o materno foram de $15,0 \%$ até os quatro meses de idade, $26,4 \%$ entre quatro e seis meses e $43,6 \%$ com mais de seis meses.

No grupo de crianças com menos de seis meses, a prevalência de mães que estavam amamentando exclusivamente seus filhos foi duas vezes maior entre aquelas que não trabalhavam fora de casa e, aproximadamente, quatro vezes maior entre aquelas que estavam em licença maternidade quando comparada com as mães que estavam trabalhando. A frequência de aleitamento materno exclusivo também foi maior entre as crianças que não usavam chupeta e que não eram primogênitas (Tabela 1).

Tabela 1. Associação entre o aleitamento materno exclusivo e as variáveis maternas, paridade e uso de chupeta nas crianças de 0 a 6 meses de vida. Porto Alegre (RS), 2008.

\begin{tabular}{|c|c|c|c|c|}
\hline & \multicolumn{4}{|c|}{ Em aleitamento materno exclusivo } \\
\hline \multicolumn{5}{|l|}{ Escolaridade materna } \\
\hline Sem ensino fundamental completo & 73 & 39,9 & 1 & - \\
\hline Ensino fundamental completo & 69 & 39,0 & $0,96(0,62-1,50)$ & \\
\hline Ensino médio completo & 58 & 41,4 & $1,07(0,66-1,71)$ & \\
\hline \multicolumn{5}{|l|}{ Trabalho materno atual } \\
\hline Trabalha fora & 25 & 26,0 & 1 & 1 \\
\hline Não trabalha fora & 136 & 42,6 & $2,11(1,24-3,62)$ & $2,05(1,20-3,48)$ \\
\hline Licença-maternidade & 52 & 57,1 & $3,79(1,96-7,37)$ & $3,81(2,00-7,27)$ \\
\hline \multicolumn{5}{|l|}{ Aleitamento na $1^{a}$ hora de vida } \\
\hline Não & 142 & 37,8 & 1 & - \\
\hline Sim & 70 & 42,9 & $1,24(0,84-1,83)$ & \\
\hline \multicolumn{5}{|l|}{ Primeiro filho } \\
\hline Sim & 76 & 33,5 & 1 & 1 \\
\hline Não & 138 & 48,8 & $1,89(1,30-2,76)$ & $2,07(1,41-3,04)$ \\
\hline
\end{tabular}

*Análise bruta; ** Análise ajustada; OR: Odds Ration; IC: Intervalo de Confiança.

Tabela 2. Proporção de crianças com idades a partir dos 6 meses que consumiram biscoito/salgadinho, açúcar/mel e café no dia anterior segundo relato da mãe. Porto Alegre (RS), 2008.

\begin{tabular}{|c|c|c|c|c|c|c|c|c|c|}
\hline & \multicolumn{3}{|c|}{ Biscoito/salgadinho } & \multicolumn{3}{|c|}{ Açúcar/mel } & \multicolumn{3}{|c|}{ Café } \\
\hline & $\mathrm{n}$ & $\%$ & OR (IC95\%) & $\mathrm{n}$ & $\%$ & OR (IC95\%) & $\mathrm{n}$ & $\%$ & OR (IC95\%) \\
\hline \multicolumn{10}{|c|}{ Escolaridade materna } \\
\hline Sem EF completo & 121 & 80,1 & 1 & 80 & 52,6 & 1 & 20 & 13,2 & 1 \\
\hline EF completo & 111 & 79,9 & $0,98(0,53-1,82)$ & 65 & 46,8 & $0,79(0,49-1,29)$ & 12 & 8,6 & $0,62(0,27-1,41)$ \\
\hline EM completo & 75 & 59,5 & $0,36(0,21-0,64)$ & 59 & 46,8 & $0,79(0,48-1,31)$ & 3 & 2,4 & $0,16(0,04-0,59)$ \\
\hline ES completo & 15 & 48,4 & $0,23(0,10-0,56)$ & 9 & 29,0 & $0,37(0,15-0,91)$ & - & - & - \\
\hline \multicolumn{10}{|c|}{ Trabalho materno atual } \\
\hline Não trabalha fora & 199 & 74,8 & 1 & 135 & 50,8 & 1 & 26 & 9,7 & 1 \\
\hline Trabalha fora & 95 & 66,4 & $0,66(0,41-1,06)$ & 66 & 45,8 & $0,81(0,53-1,26)$ & 7 & 4,9 & $0,47(0,18-1,17)$ \\
\hline
\end{tabular}

EF: Ensino Fundamental; EM: Ensino Médio; ES: Ensino Superior; OR: Odds Ration; IC: Intervalo de Confiança. 
436 | PDB CAMPAGNOLO et al.

Maior nível de escolaridade materno foi associado ao menor consumo de biscoito/salgadinho, açúcar/mel, café e feijão, e maior frequência de consumo de legumes/verduras e carne entre os lactentes (Tabelas 2, 3 e 4). Entre os lactentes das mães que não trabalhavam fora de casa, a frequência de consumo de suco de fruta, legumes/ verduras, fruta e feijão foi menor quando comparada com os lactentes das mães que trabalhavam fora de casa (Tabelas 3 e 4).

\section{I S C U S S Ã O}

Observou-se evolução significativa dos indicadores de aleitamento materno na cidade de Porto Alegre entre 1999 e $2008^{\circ}$. Apesar disso, os dados ainda estão distantes dos parâmetros preconizados e observou-se neste estudo que o uso de chupeta e trabalho materno estiveram implicados nesse processo. Não ter ocupação fora do lar e menor escolaridade materna foram associados ao consumo de alimentos de baixo valor nutricional entre 6 e 12 meses de vida.

A prevalência de AME foi maior entre os lactentes cujas mães não trabalhavam fora de casa e estavam em licença maternidade, evidenciando o fato de que a separação do filho devido à volta da mãe ao trabalho fora do lar é fator de risco independente para a introdução precoce de outros líquidos e alimentos, com repercussões óbvias na saúde da criança ${ }^{12}$. O crescimento da participação feminina no mercado de trabalho no país foi uma das transformações sociais mais mar-

Tabela 3. Proporção de crianças com idades a partir dos 6 meses que consumiram refrigerante, suco industrializado e suco de fruta no dia anterior segundo relato da mãe. Porto Alegre (RS), 2008.

\begin{tabular}{|c|c|c|c|c|c|c|c|c|c|}
\hline & \multicolumn{3}{|c|}{ Refrigerante } & \multicolumn{3}{|c|}{ Suco industrializado } & \multicolumn{3}{|c|}{ Suco de fruta } \\
\hline & $\mathrm{n}$ & $\%$ & OR (IC95\%) & $\mathrm{n}$ & $\%$ & OR (IC95\%) & $n$ & $\%$ & OR (IC95\%) \\
\hline \multicolumn{10}{|c|}{ Escolaridade materna } \\
\hline Sem EF completo & 24 & 15,8 & 1 & 21 & 13,9 & 1 & 81 & 54,0 & 1 \\
\hline EF completo & 26 & 18,8 & $1,24(0,64-1,82)$ & 20 & 14,6 & $1,06(0,52-2,15)$ & 83 & 61,0 & $1,33(0,81-2,20)$ \\
\hline EM completo & 3 & 10,2 & $0,61(0,28-1,32)$ & 10 & 7,9 & $0,53(0,22-1,25)$ & 79 & 63,2 & $1,46(0,88-2,45)$ \\
\hline ES completo & - & - & - & 3 & 9,7 & $0,66(0,15-2,58)$ & 22 & 71,0 & $2,08(0,84-5,26)$ \\
\hline \multicolumn{10}{|c|}{ Trabalho materno atual } \\
\hline Não trabalha fora & 47 & 17,7 & 1 & 34 & 12,8 & 1 & 143 & 54,6 & 1 \\
\hline Trabalha fora & 13 & 9,0 & $0,46(0,22-0,92)$ & 19 & 13,3 & $1,04(0,54-1,96)$ & 97 & 67,8 & $1,75(1,12-2,77)$ \\
\hline
\end{tabular}

EF: Ensino Fundamental; EM: Ensino Médio; ES: Ensino Superior; OR: Odds Ration; IC: Intervalo de Confiança.

Tabela 4. Proporção de crianças com idades a partir dos 6 meses que consumiram suco de frutas, legumes/verduras, carne, fruta e feijão no dia anterior segundo relato da mãe. Porto Alegre (RS), 2008.

\begin{tabular}{|c|c|c|c|c|c|c|c|c|c|c|c|c|}
\hline & \multicolumn{3}{|c|}{ Legumes/verduras } & \multicolumn{3}{|c|}{ Carne } & \multicolumn{3}{|r|}{ Fruta } & \multicolumn{3}{|r|}{ Feijão } \\
\hline & $n$ & $\%$ & OR (IC95\%) & $n$ & $\%$ & OR (IC95\%) & $n$ & $\%$ & OR (IC95\%) & $n$ & $\%$ & OR (IC95\%) \\
\hline \multicolumn{13}{|c|}{ Escolaridade materna } \\
\hline Sem EF completo & 112 & 80,6 & 1 & 96 & 69,6 & 1 & 121 & 81,2 & 1 & 117 & 84,2 & 1 \\
\hline EF completo & 104 & 81,9 & $1,09(0,56-2,11)$ & 89 & 69,5 & $1,00(0,57-17,4)$ & 113 & 81,3 & $1,01(0,53-1,89)$ & 97 & 75,8 & $0,59(0,31-1,13)$ \\
\hline EM completo & 118 & 95,9 & $5,69(1,99-17,49)$ & 101 & 82,8 & $2,10(1,12-3,98)$ & 111 & 88,1 & $1,71(0,83-3,57)$ & 90 & 73,2 & $0,51(0,27-0,98)$ \\
\hline ES completo & 29 & 100,0 & & 24 & 82,8 & $2,10(0,70-6,77)$ & 28 & 90,3 & $2,16(0,57-9,61)$ & 16 & 57,1 & $0,25(0,10-0,66)$ \\
\hline \multicolumn{13}{|l|}{ Trabalho materno } \\
\hline Não trabalha fora & 204 & 82,3 & 1 & 179 & 72,2 & 1 & 208 & 78,2 & 1 & 191 & 76,7 & 1 \\
\hline Trabalha fora & 124 & 92,5 & $2,7(1,25-5,80)$ & 107 & 80,5 & $1,58(0,92-2,70)$ & 134 & 93,7 & $4,16(1,90-9,09)$ & 105 & 78,9 & $1,69(1,01-2,85)$ \\
\hline
\end{tabular}

EF: Ensino Fundamental; EM: Ensino Médio; ES: Ensino Superior; OR: Odds Ration; IC: Intervalo de Confiança. 
cantes ocorridas nos últimos 40 anos. Pesquisas nacionais mostram que esse fenômeno é cada vez mais intenso e sem tendências de retrocesso, implicando mudança do comportamento em relação à amamentação ${ }^{13}$. Dessa forma, ressalta-se a importância do amparo legal às mães trabalhadoras, com garantia de licença maternidade remunerada, disponibilização de creche, locais apropriados para ordenha e estoque do leite e flexibilização da jornada com pausas para amamentação ${ }^{12}$.

O fato de a frequência de AME ter sido maior entre aqueles que não eram primogênitos pode estar atrelado à insegurança e à falta de preparo da mãe para cuidar do primeiro filho ${ }^{14}$. A interpretação equivocada do choro do bebê, com a crença de que o leite não supre de forma integral suas necessidades, pode implicar a introdução de aleitamento artificial, uso de mamadeiras e consequente diminuição da sucção dos mamilos e produção de leite ${ }^{15}$. Estudo realizado em São Paulo também evidenciou maior consumo de refeições lácteas em crianças de 6 a 12 meses de mães primíparas $^{16}$.

A última pesquisa nacional sobre a prevalência de aleitamento materno revelou que, no tocante à comparação entre as capitais, a pior situação em relação ao uso da chupeta em crianças menores de 12 meses foi verificada em Porto Alegre ${ }^{17}$. Há evidências de que o padrão de movimentação da musculatura facial e a dinâmica de sucção das crianças que utilizam chupetas e bicos são diferentes daquelas que sugam apenas o peito ${ }^{18}$. Há alguns anos, foi descrito o fenômeno da "confusão de bicos", em que a criança em uso de bicos artificiais diminui a eficiência da sucção no seio, reduzindo a produção do leite e aumentando a chance da oferta precoce de alimentos pela mãe ${ }^{19}$. Alguns estudos nacionais já descreveram associação entre o uso da chupeta e a interrupção precoce do aleitamento materno exclusivo $^{20-23}$. O delineamento transversal do presente estudo, no entanto, limita o estabelecimento de relação causal. O'Connor et al. ${ }^{24}$, em revisão sistemática de ensaios clínicos randomizados, apresentaram fortes evidências de que a chupeta seria introduzida como consequência de problemas na técnica ou na redução da motivação para amamentar, não sendo a causa direta da introdução de alimentos. Os motivos mais frequentes pelos quais as mães oferecem chupeta a seu primeiro filho foram a necessidade de acalmar o bebê e de fazê-lo dormir ${ }^{25}$, e essas razões podem ser mais evidentes em mães menos experientes.

Os dados apresentados descrevem alta prevalência de consumo precoce de açúcar, doce, refrigerante e petisco salgado. A maior parte desses alimentos contém alta densidade energética com pouca quantidade de micronutrientes e pode ser indicador de hábitos alimentares inadequados na adolescência e na vida adulta26,27.

Os resultados do presente trabalho vão ao encontro dos achados apresentados por diversos autores que afirmam que o hábito allmentar da criança é fortemente influenciado por características maternas e familiares ${ }^{28-30}$. Os lactentes cujas mães possuíam emprego fora de casa apresentaram maior frequência de consumo de vegetais e leguminosas. Com exceção do feijão, o maior nível de escolaridade foi associado ao menor consumo de alimentos considerados impróprios para essa faixa etária, pois essas mulheres possivelmente têm maior acesso às informações sobre práticas alimentares saudáveis ${ }^{31}$. Esse aspecto pode ser observado no trabalho realizado por Corrêa et al. ${ }^{32}$, na cidade de Florianópolis (SC), em que as mães com menos de oito anos de estudo apresentaram o dobro de chance de oferecerem alimentos do grupo dos doces (açúcar de adição, bolachas, pudins e gelatinas) para os filhos. Em Pelotas (RS), foram observados alto consumo energético e introdução precoce de leite de vaca especialmente para crianças de menor nível socioeconômico ${ }^{33}$. Estudo internacional também demonstrou que os anos de estudo da mãe foram inversamente associados ao consumo de bebidas açucaradas, sobremesas e doces pelas crianças pequenas ${ }^{34}$. O maior nível educacional e o trabalho fora de casa também podem estar relacionados à renda familiar mais elevada, o que possibilita maior acesso aos alimentos de custo mais alto, como vegetais e carnes. 
O estudo apresenta algumas limitações que merecem comentários. Por ser um estudo transversal, não é possível avaliar a relação causaefeito entre as variáveis. Outro aspecto é a amostragem não probabilística por conveniência, a qual poderia levar a vieses. Porém, a realização de pesquisas durante as campanhas de vacinação é uma estratégia do governo brasileiro tendo em vista sua facilidade e seu menor custo e principalmente levando em consideração a ampla cobertura nos últimos anos, o que torna a amostra representativa da cidade de Porto Alegre. Variáveis relacionadas à gestação e às condições de nascimento, que poderiam interferir nos desfechos investigados, não foram medidas. Ainda assim, os resultados são relevantes uma vez que poucos estudos nacionais têm abordado a introdução de alimentos de alta densidade energética e baixa densidade nutricional no primeiro ano de vida, servindo como gerador de hipóteses para futuras investigações mais detalhadas.

\section{O N CLUS Ã O}

Os resultados apresentados indicam que, embora tenha ocorrido progresso nos índices de aleitamento materno exclusivo nos últimos 10 anos na cidade de Porto Alegre, os valores ainda estão muito aquém da recomendação. Os avanços são decorrentes das inúmeras iniciativas em prol do aleitamento materno e da alimentação saudável que vêm sendo implementadas desde o início da década de 1980 com o Programa Nacional de Incentivo ao Aleitamento Materno e na década de 1990 com o Hospital Amigo da Criança, e, recentemente, com a implementação da Rede Amamenta Brasil e Iniciativa Unidade Básica Amiga da Amamentação. Porém, o desafio permanece e a promoção da educação permanente para os profissionais da saúde com abordagem prioritária nas deficiências encontradas necessita ser ampliada para que as estratégias sejam efetivamente implementadas na atenção primária à saúde. Entretanto, alguns grupos populacionais podem ser priorizados pelos programas de incentivo à amamentação e à alimentação saudável. Mães primíparas e que trabalham fora de casa constituem categorias de risco para interrupção precoce do AME; além disso, o uso da chupeta deve ser interpretado, pelos profissionais da saúde, como um indicativo de problemas na amamentação. Mães com menor escolaridade e que não trabalham fora de casa apresentaram maiores riscos para a introdução de alimentos de baixo valor nutricional de forma precoce, prática que pode gerar consequências negativas no estado nutricional e na saúde das crianças.

\section{COLABORADORES}

PDB CAMPAGNOLO participou da análise, da interpretação dos resultados e da elaboração do artigo. MLC LOUZADA participou da interpretação dos resultados e da elaboração do artigo. EL SILVEIRA participou da coordenação do projeto de pesquisa, da análise, da interpretação dos resultados e da elaboração do artigo. MR VITOLO participou da concepção das análises dos dados, da interpretação dos resultados e da elaboração do artigo.

\section{REFERÊ NCIAS}

1. Brasil. Ministério da Saúde. Dez passos para uma alimentação saudável: guia alimentar para crianças menores de dois anos. Brasília (DF): MS; 2002.

2. Beauchamp GK, Moran M. Acceptance of sweet and salty tastes in 2-year-old children. Appetite. 1984; 5(4):291-305.

3. Skinner JD, Carruth BR, Wendy B, Ziegler PJ. Children's food preferences: a longitudinal analysis. J Am Diet Assoc. 2002; 102(11):1638-47.

4. Leathwood P, Maier A. Early influences on taste preferences. Nestle Nutr Workshop Ser Pediatr Program. 2005; 56:127-38.

5. Shim JE, Kim J, Mathai RA, Strong Kids Research Team. Associations of infant feeding practices and picky eating behaviors of preschool children. J Am Diet Assoc. 2011; 111(9):1363-8.

6. Gale C, Logan KM, Santhakumaran S, Parkinson JR, Hyde MJ, Modi N. Effect of breastfeeding compared with formula feeding on infant body composition: a systematic review and metaanalysis. Am J Clin Nutr. 2012; 95(3):656-69.

7. Socha P, Grote V, Gruszfeld D, Janas R, Demmelmair $\mathrm{H}, \mathrm{Closa}-$ Monasterolo R, et al. Milk protein intake, the metabolic-endocrine response, and growth in 
infancy: data from a randomized clinical trial. Am J Clin Nutr. 2011; 94(6 Suppl):1776S-84S.

8. Brasil. Ministério da Saúde. Pesquisa nacional de demografia e saúde da criança e da mulher. Brasília: MS; 2008.

9. Venancio SI, Escuder MM, Saldiva SR, Giugliani ER. Breastfeeding practice in the Brazilian capital cities and the Federal District: current status and advances. Pediatrics. 2010; 86(4):317-24.

10. Silva LMP, Venâncio SI, Marchioni DML. Práticas de alimentação complementar no primeiro ano de vida e fatores associados. Rev Nutr. 2010; 23(6):983-92. doi: 10.1590/S1415-52732010000600005.

11. World Health Organization. Global strategy for infant and young child feeding. Geneva: WHO; 2003.

12. Chuang $\mathrm{CH}$, Chang PJ, Chen YC, Hsieh WS, Hurng BS, Lin SJ, et al. Maternal return to work and breastfeeding: a population-based cohort study. Int J Nurs, Stud. 2010; 47(4):461-74.

13. Araujo OD, Cunha AL, Lustosa LR, Nery IS, Mendonca RC, Campelo SM. Aleitamento materno: fatores que levam ao desmame precoce. Rev Bras Enferm. 2008; 61(4):488-92.

14. Cronin C, McCarthy G. First-time mothers: identifying their needs, perceptions and experiences. J Clin Nurs. 2003; 12(2):260-7.

15. Giugliani ER, Rocha VL, Neves JM, Polanczyk CA, Seffrin CF, Susin LO. Conhecimentos maternos em amamentacao e fatores associados. J Pediatr. 1995; 71(2):77-81.

16. Saldiva SRDM, Escuder MM, Mondini L, Levy RB, Venancio SI. Práticas alimentares de crianças de 6 a 12 meses e fatores maternos associados. J Pediatr. 2007; 83(1):53-8.

17. Brasil. Ministério da Saúde. II Pesquisa de prevalência de aleitamento materno nas capitais brasileiras e Distrito Federal. Brasília: MS; 2009.

18. Organização Mundial da Saúde. Evidências científicas dos dez passos para o sucesso no aleitamento materno. Brasília: Organização Pan-Americana de Saúde; 2001.

19. Neifert M, Lawrence R, Seacat J. Nipple confusion: toward a formal definition. J Pediatr. 1995; 126(6): 125-9.

20. Feldens CA, Vitolo MR, Rauber F, Cruz LN, Hilgert JB. Risk factors for discontinuing breastfeeding in southern Brazil: a survival analysis. Matern Child Health. J. 2011; 16(6):1257-65.

21.Zapana PM, Oliveira MN, Taddei JA. Factors determining the breastfeeding in children attending public and not-for-profit daycare centers in São Paulo, Brazil. Arch Latinoam Nutr. 2010; 60(4): 360-7.

22. Vieira GO, Martins CC, Vieira TO, Oliveira NF, Silva LR. Factors predicting early discontinuation of exclusive breastfeeding in the first month of life. J Pediatr. 2010; 86(5):441-4.

23. Sanches MT, Buccini GD, Gimeno SG, Rosa TE, Bonamigo AW. Fatores associados a interrupção do aleitamento materno exclusivo de lactentes nascidos com baixo peso assistidos na atenção básica. Cad Saúde Pública. 2011; 27(5):953-65.

24. O'Connor NR, Tanabe KO, Siadaty MS, Hauck FR. Pacifiers and breastfeeding: a systematic review. Arch Pediatr Adolesc Med. 2009; 163(4):378-82.

25. Mauch CE, Scott JA, Magarey AM, Daniels LA. Predictors of and reasons for pacifier use in firsttime mothers: an observational study. BMC Pediatr. 2012;12:7

26. Fiorito LM, Marini M, Mitchell DC, Smiciklas-Wright H, Birch LL. Girls' early sweetened carbonated beverage intake predicts different patterns of beverage and nutrient intake across childhood and adolescence. J Am Diet Assoc. 2010; 110(4): 543-50.

27. Northstone K, Emmett PM. Are dietary patterns stable throughout early and mid-childhood? A birth cohort study. Br J Nutr. 2008; 100(5):1069-76.

28. Robinson S, Marriott L, Poole J, Crozier S, Borland $\mathrm{S}$, Lawrence $\mathrm{W}$, et al. Dietary patterns in infancy: the importance of maternal and family influences on feeding practice. Br J Nutr. 2007; 98(5): 1029-37.

29. Ventura AK, Birch LL. Does parenting affect children's eating and weight status? Int J Behav Nutr Phys Act. 2008; 5:15.

30. Damião JJ. Influência da escolaridade e do trabalho maternos no aleitamento materno exclusivo. Rev Bras Epidemiol. 2008; 11(3):442-52.

31. Skafida $\mathrm{V}$. The relative importance of social class and maternal education for breast-feeding initiation. Public Health Nutr. 2009; 12(12):2285-92.

32. Corrêa NP, Corso ACT, Moreira EAM, Kazapi IAM. Alimentação complementar e características maternas de crianças menores de dois anos de idade em Florianópolis (SC). Rev Paul de Pediatr. 2009; 27(3): 258-64.

33. Romulus-Nieuwelink JJ, Doak C, Albernaz E, Victora CG, Haisma H. Breast milk and complementary food intake in Brazilian infants according to socioeconomic position. Int J Pediatr Obes. 2011; 6(2-2): e508-14.

34. Northstone K, Rogers I, Emmett P. Drinks consumed by 18-month-old children: are current recommendations being followed? Eur J Clin Nutr. 2002; 56(3):236-44. 
